\title{
Visual search: Efficiency continuum or distinct processes?
}

\author{
NICK HASLAM, MATTHEW PORTER, and LOUIS ROTHSCHILD \\ New School for Social Research, New York, New York
}

\begin{abstract}
Whether visual search involves two distinct processes - traditionally referred to as serial and parallel-or a single process operating on a continuum of efficiency or speed, is an issue with a long history in the study of attention. On the basis of the unimodality of search slope distributions in a very large data set, Wolfe (1998) argued for a continuum model. Reanalysis of this data set using statistical procedures more appropriate for adjudicating between continuous and discontinuous models supports the existence of two distinct processes.
\end{abstract}

In a recent analysis of 2,500 visual search task sessions conducted in his laboratory, involving about one million trials, Wolfe (1998) called into question the venerable distinction between parallel and serial search. For both targetpresent and target-absent trials, and across a variety of search types, Wolfe found that the distributions of participants' search slopes - measured as reaction time divided by number of items in the display (msec/item) - were not bimodal. This finding, Wolfe argued, "provides no support for a simple, data-driven division of searches into serial and parallel (or anything else)" (p. 38). In place of this "mythical divide," Wolfe proposes that searches vary along a continuum of efficiency, consistent with other recent models of visual search that posit a single mechanism.

Wolfe's (1998) argument for a continuum model can be criticized on statistical grounds. Bimodality in an observed distribution is an unreliable criterion for deciding whether the processes that gave rise to the distribution are categorically distinct (Murphy, 1964). Two distinct but overlapping latent distributions may be admixed in an observed distribution without yielding two modes, and will in fact do so only if the overlap is minimal (separation of latent distributions $\cong$ three standard deviations). Figure 1 illustrates this point. Two normal distributions (dotted lines) separated by a substantial two standard deviations, one twice as populous as the other, yield a summed distribution (solid line) that is positively skewed but shows no evidence of a second mode. Consequently, the unimodality of Wolfe's slope distributions is ambiguous. It may, as Wolfe suggests, straightforwardly reveal a single underlying distribution of searches that vary along a continuum of speed or efficiency. Alternatively, it may conceal two dis-

The authors thank Jeremy Wolfe for permission to use his visual search data and the Air Force Office of Scientific Research for supporting its collection. We thank John Wixted and three anonymous reviewers for their thoughtful guidance. Correspondence concerning this article should be addressed to N. Haslam, Department of Psychology, New School for Social Research, 65 Fifth Avenue, New York, NY 10003 (e-mail: haslam@newschool.edu). tinct distributions - one corresponding to relatively slow searches and the other to relatively fast searches - that derive from distinct processes, whether or not these processes are best described as serial and parallel.

This ambiguity can be addressed in a statistically rigorous fashion by determining whether the slope distributions in Wolfe's (1998) data set are better modeled by a single distribution (continuum model) or by two admixed distributions (distinct process model). Commingling analysis, a variant of mixture modeling (Everitt \& Hand, 1981), is an established procedure for doing this. As performed by the SKUMIX algorithm (MacLean, Morton, Elston, \& Yee, 1976), commingling analysis fits a series of models to a data set. For the basic 1-distribution model, two "free" parameters are adjusted to maximize fit, the overall mean $(U)$ and within-component variance $(E)$. For the basic 2-distribution model, in contrast, four parameters are adjusted: $U, E$ (constrained to be the same for both component distributions), the separation of the two distributions $(T)$, and the proportion of the population in the higher-scoring distribution $(Q)$. A skewness parameter $(P)$ can also be varied so that the component distributions are skewed by an adjustable power transform (constrained to be the same for both distributions in the 2-distribution case). By this means, skewed 1- and 2-distribution models, with three and five free parameters, respectively, can also be tested.

Using maximum likelihood estimation, the SKUMIX algorithm iterates the various parameters of these four models in order to make the observed data most likely to have occurred given the particular model being fitted. This likelihood, denoted by $L$, yields the fit statistic $-2 \ln L(-2$ times the $\log$ of $L$ ). The fit of alternative models can be statistically compared because the difference between the values of this statistic for the respective models is distributed as chi square with degrees of freedom equal to the difference in their number of free parameters. For example, comparing the skewed 2-distribution model with the unskewed 1-distribution model would involve three (i.e., $5-2$ ) degrees of freedom. 


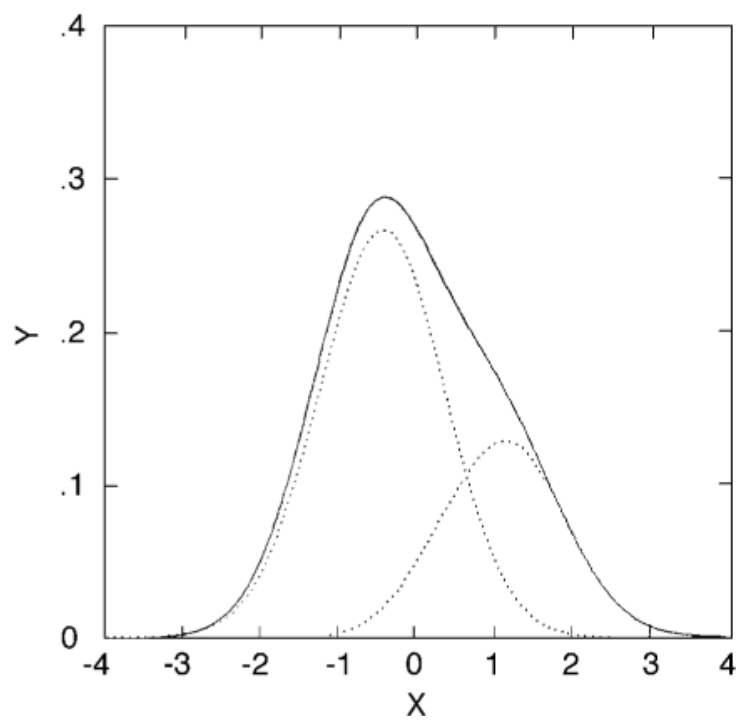

Figure 1. Skewed admixture distribution (solid line) representing the summation of two latent distributions (dotted lines) of unequal proportions.

If the commingling analyses indicate that 2-distribution models yield superior fit to the search slope data, as compared with 1-distribution models, they would imply that the unimodality of Wolfe's (1998) search slopes are not best explained by a single process that gives rise to a continuum of search efficiency. To infer the existence of distinct search processes with any confidence, however, it is desirable not only that the 2-distribution models yield superior fit but also that their parameters support several additional corollaries of a distinct process model. These consistency tests depend on values of the $Q$ parameter (i.e., the proportion of slopes in the slower distribution).

First, if the search slope distributions truly derive from distinct processes, the commingling analyses should generate similar estimates of $Q$ across various transformations of the data set. If the data set contains two admixed distributions, the proportions of these distributions should not vary, whether the data are raw or rescaled by logarithmic, power, or other transformations, because they represent real properties of the latent situation rather than being artifacts of measurement. If the analyses are not robust in this way, the superior fit of the 2-distribution models might simply be an artifact of the particular distributional form of the raw data.

Second, if 2-distribution models are to be validated, they should yield higher estimates of $Q$ for the target-absent searches than for the target-present searches. The former are substantially slower, as Wolfe (1998) showed, and a larger proportion of them should therefore belong to the slower search distribution.

Third, because certain types of search task are quicker than others, they should tend to yield relatively low $Q$ estimates. If there are indeed two distinct search processes, the slower process should be employed less frequently in these quicker tasks. In Wolfe's (1998) data set, feature searches were quicker on average than conjunction searches, which in turn were quicker than spatial configuration searches, and $Q$ estimates in the 2-distribution models should follow the same ordering if these models are to be fully supported.

Fourth, the 2-distribution models should, ideally, suggest a similar placement of the threshold between the distributions produced by the faster and slower search processes in the various search types. If there are two distinct processes that operate in visual search, their respective search slope distributions should have more or less equivalent locations (i.e., mean speeds) across search tasks, even if people are more likely to employ a particular process in some tasks than in others. Consequently, the slope marking the best threshold for separating the two distributions should be stable across tasks.

\section{METHOD}

We used the SKUMIX algorithm to perform commingling analyses of the distributions of the 2,500 target-presen t and the 2,500 targetabsent search slopes analyzed by Wolfe (1998). Analyses were conducted for the entire set of search slopes and separately for each of the distinct search types included in the set (conjunction, $n=1,218$; feature, $n=490$; miscellaneous, $n=325$; spatial configuration, $n=205$; and within-dimension conjunction, $n=182$ ), except for the hard feature, search type, whose sample size $(n=80)$ afforded insufficient statistical power. Each analysis was conducted with three versions of the slope distributions to test the robustness of the findings with respect to distributional assumptions. Analyses were performed first for the highly skewed raw slope distributions (e.g., Wolfe, 1998, Figure 1) and then for skew-reducing log- and power-transformed (square root) versions of these distributions. Because a small minority of search slopes was negative ( $\min =-19.4)$, a constant $(25)$ was added to all slopes so that the transformed values would be determinate. In sum, 36 separate commingling analyses were performed ( 2 target conditions $\times 6$ search types $\times 3$ distributional forms).

Following established procedures, four distinct models were fit for each commingling analysis: 1- and 2-distribution models with the component distributions either constrained to normality or free to show skewness. For each of the 36 analyses, the best-fitting 1and 2-distribution models were first selected by determining whether freeing the skew parameter $P$ improved fit over the respective unskewed model, which was retained in the absence of significant improvement. The best-fitting 2-distribution model was then compared with the best-fitting 1-distribution model. Conservatively, the 1-distribution model was retained when no significant improvement was found.

\section{RESULTS}

Results of the commingling analyses are summarized in Tables 1 and 2. Table 1 presents the comparisons of model fit for the 1- and 2-distribution models, and Table 2 presents estimates of the $Q$ parameter from the superiorfitting 2-distribution models (i.e., the proportion of search slopes in the slower distribution). Most of the analyses (32 of 36) strongly favor the 2-distribution models, which implies the existence of distinct search processes. The exceptions are most of the spatial configuration search analyses. These exceptions occur in a slow search type that is, as Wolfe (1998) notes, a "gold standard" for serial search, suggesting that a 1-distribution model should yield a good 
fit to the slope distributionseven if there are distinct search processes resembling parallel and serial mechanisms. Consequently, the findings for the spatial configuration search type are not inconsistent with the overall pattern of findings.

Figures $2 \mathrm{~A}$ and $2 \mathrm{~B}$ illustrate the results of the commingling analysis in the case of the log-transformed total sample target-present data. Both figures present a histogram of the search slope distribution overlaid by a curve representing the fitted model. Figure $2 \mathrm{~A}$ shows the bestfitting 1-distribution model, which incorporates positive skew, and Figure 2B shows the best-fitting 2-distribution model, an admixture of two skewed distributions. The model in Figure 2B clearly provides a close and superior fit $(p<.0001)$, established by submitting the difference in fit values for the respective models to a chi square test with $d f$ equivalent to the difference in their free parameters (MacLean et al., 1976).

The $Q$ parameter estimates presented in Table 2 offer further support for the validity of these findings in regard to the four consistency tests presented above. First, the estimates for each search type show substantial convergence across the three distribution types, despite their different scalings and levels of skewness. The mean spread of the three estimates for the 10 relevant data sets $(5$ search types [excluding spatial configuration] $\times 2$ target conditions) was only 0.07 , suggesting that the 2 -distribution models are robust across data transformations. Second, in 14 of the 15 target-present/target-absent pairs, the estimates show a higher proportion of slower searches in the target-absent conditions, consistent with the validity of the distinct process model. Third, the $Q$ estimates indicate higher rates of slower search in the slower search types (i.e., conjunction searches $>$ feature searches).
To assess the fourth consistency test- convergence in the location of the threshold between the two apparently distinct distributions across search types-we used the $Q$ estimates to estimate the optimal search slope for dividing the distributions and allocating individual slopes to their correct distribution. Meehl (1995) shows that the best threshold is to be found where the latent distributions intersect. This, in turn, can be approximated by locating the point above which the slower distribution's proportion of slopes (i.e., $Q$ ) occurs. If $25 \%$ of the slopes in the sample belong to the slower distribution (i.e., $Q=0.25$ ), for example, a good estimate of the optimal threshold would be the search slope at the sample's 75 th percentile. Given the overlap of the two distributions (cf. Figure 1), some of the slopes in the sample's slowest $25 \%$ will belong to the tail of the faster distribution, just as some slopes in the sample's fastest $75 \%$ will belong to the tail of the slower distribution. However, these tails will be of similar size and constitute the threshold's unavoidableidentification errors.

In the manner described here, we derived estimates of the threshold point from each of the five search types (excluding spatial configuration searches) that were best fit by the 2-distribution models, and separately for the targetpresent and target-absent searches, yielding 10 estimates. For each estimate, we took the mean estimate of $Q$ across the three distribution types and located the search slope corresponding to it (i.e., the $[Q-1] * 100$ percentile). The 10 estimates were quite convergent, ranging between 7.8 and $14.7 \mathrm{msec} / \mathrm{item}$, consistent with the task independence of the search processes presumed to underlie the two distributions. Notably, the mean estimate was $10.7 \mathrm{msec} / \mathrm{item}$, strikingly close to the informal $10 \mathrm{msec} /$ item threshold that is sometimes used for dividing parallel and serial search tasks.

Table 1

Chi Square Values for Improvement in Fit Afforded

by Best-Fitting 2-Distribution Model Over

Best-Fitting 1-Distribution Model ( $d f$ in Parentheses)

\begin{tabular}{lrlrr}
\hline \multicolumn{1}{c}{ Search Type } & $N$ & Distribution Type & Target-Present & Target-Absent \\
\hline Total sample & 2,500 & Untransformed & $217.89(2) \ddagger$ & $284.18(2) \ddagger$ \\
& 2,500 & Log transformed & $61.43(2) \ddagger$ & $59.11(2) \ddagger$ \\
Conjunction & 2,500 & Power transformed & $96.83(2) \ddagger$ & $121.71(2) \ddagger$ \\
& 1,218 & Untransformed & $75.74(2) \ddagger$ & $530.03(2) \ddagger$ \\
Feature & 1,218 & Log transformed & $29.26(2) \ddagger$ & $29.29(2) \ddagger$ \\
& 1,218 & Power transformed & $32.05(2) \ddagger$ & $41.92(2) \ddagger$ \\
Miscellaneous & 490 & Untransformed & $37.34(2) \ddagger$ & $72.78(2) \ddagger$ \\
& 490 & Log transformed & $55.02(1) \ddagger$ & $115.11(2) \ddagger$ \\
Spatial configuration & 490 & Power transformed & $45.94(2) \ddagger$ & $85.70(2) \ddagger$ \\
& 325 & Untransformed & $51.32(2) \ddagger$ & $26.62(2) \ddagger$ \\
& 325 & Log transformed & $16.45(2) \dagger$ & $10.82(2) * *$ \\
Within-dimension & 325 & Power transformed & $78.75(2) \ddagger$ & $13.54(2) * *$ \\
conjunction & 205 & Untransformed & $4.60(1) *$ & $2.45(2)$ \\
& 205 & Log transformed & $4.17(2)$ & $4.75(3)$ \\
& 182 & Power transformed & $3.23(2)$ & $5.96(1)^{*}$ \\
& 182 & Log transformed & $22.25(2) \ddagger$ & $22.71(2) \ddagger$ \\
\hline
\end{tabular}

${ }^{*} p<.05 . \quad * * p<.01 . \quad \dagger p<.001 . \quad{ }^{\ddagger} p<.0001$. 
Table 2

Estimates of Parameter $Q$ (Proportion of Slower Distribution Searches) From Best-Fitting 2-Distribution Models

\begin{tabular}{llcc}
\hline $\begin{array}{c}\text { Search } \\
\text { Type }\end{array}$ & \multicolumn{1}{c}{$\begin{array}{c}\text { Distribution } \\
\text { Type }\end{array}$} & $\begin{array}{c}\text { Target- } \\
\text { Present }\end{array}$ & $\begin{array}{c}\text { Target- } \\
\text { Absent }\end{array}$ \\
\hline Total sample & Untransformed & .52 & .62 \\
& Log transformed & .47 & .59 \\
Conjunction & Power transformed & .51 & .65 \\
& Untransformed & .62 & .52 \\
Feature & Log transformed & .45 & .65 \\
& Power transformed & .48 & .64 \\
Miscellaneous & Untransformed & .34 & .39 \\
& Log transformed & .32 & .37 \\
& Power transformed & .32 & .36 \\
Spatial configuration & Untransformed & .50 & .62 \\
& Log transformed & .47 & .80 \\
& Power transformed & .49 & .74 \\
Within-dimension & Untransformed & .21 & - \\
conjunction & Log transformed & - & - \\
& Power transformed & - & .15 \\
& Untransformed & .64 & .69 \\
\hline
\end{tabular}

\section{DISCUSSION}

The commingling analyses strongly suggest that despite their unimodality, Wolfe's (1998) search data are not well explained by a continuum model. Two-distribution models, implying distinct fast and slow search processes, yielded substantially better fit to the search slope data than did the 1-distribution models in almost every analysis. Moreover, four additional tests were consistent with the robustness and credibility of the distinct process account.

Although they clearly weigh against a simple continuum view, the implications of these reanalyses for models of visual search are not straightforward. There appear to be several possibilities. First, the findings might suggest reinstating or strengthening the parallel/serial distinction. Second, some form of integration of continuum and distinct process models might be required. Third, the conclusiveness of the findings against the continuum model might be disputed.

The first possible implication of the findings is an unattractive one. As Wolfe (1998) and others have noted, most recent models of visual search (e.g., Duncan \& Humphries, 1989; Nakayama, 1990; Treisman, 1993) have abandoned the parallel/serial distinction, which does not appear to do justice to the complexity of empirical findings. The findings we report do not directly support this distinction in any case, referring only to the existence of distinct processes and not to how they should be characterized. Our suggestion is therefore not that the parallel/serial distinction should be resuscitated, but that the idea of distinct search processes should not be abandoned as it has been in the move toward continuum models.

The second alternative, proposing an integration of continuum and distinct process accounts, is more appealing. Our findings may complicate single mechanism or continuum models, but not refute them. The superiority of 2-distribution models does not, after all, entail the existence of two entirely distinct, separate, or pure underlying mechanisms. There may be no such simple mapping between processes or mechanisms and performance outputs, and several more complex models could also produce categorically distinct slope distributions.

First, a single search mechanism may be employed to varying degrees along an efficiency or speed continuum but have two "settings" to which it gravitates. By imperfect analogy, horses can move at a continuous variety of speeds but naturally fall into speed ranges defined by walking, trotting, cantering, and so forth. Second, a single search process could operate along a speed or efficiency continuum but have a threshold of some sort, above which it operates in a markedly more or less efficient way (e.g., a spring's resistance to a pulling force below vs. above its "elastic limit"). Third, two distinct search processes that
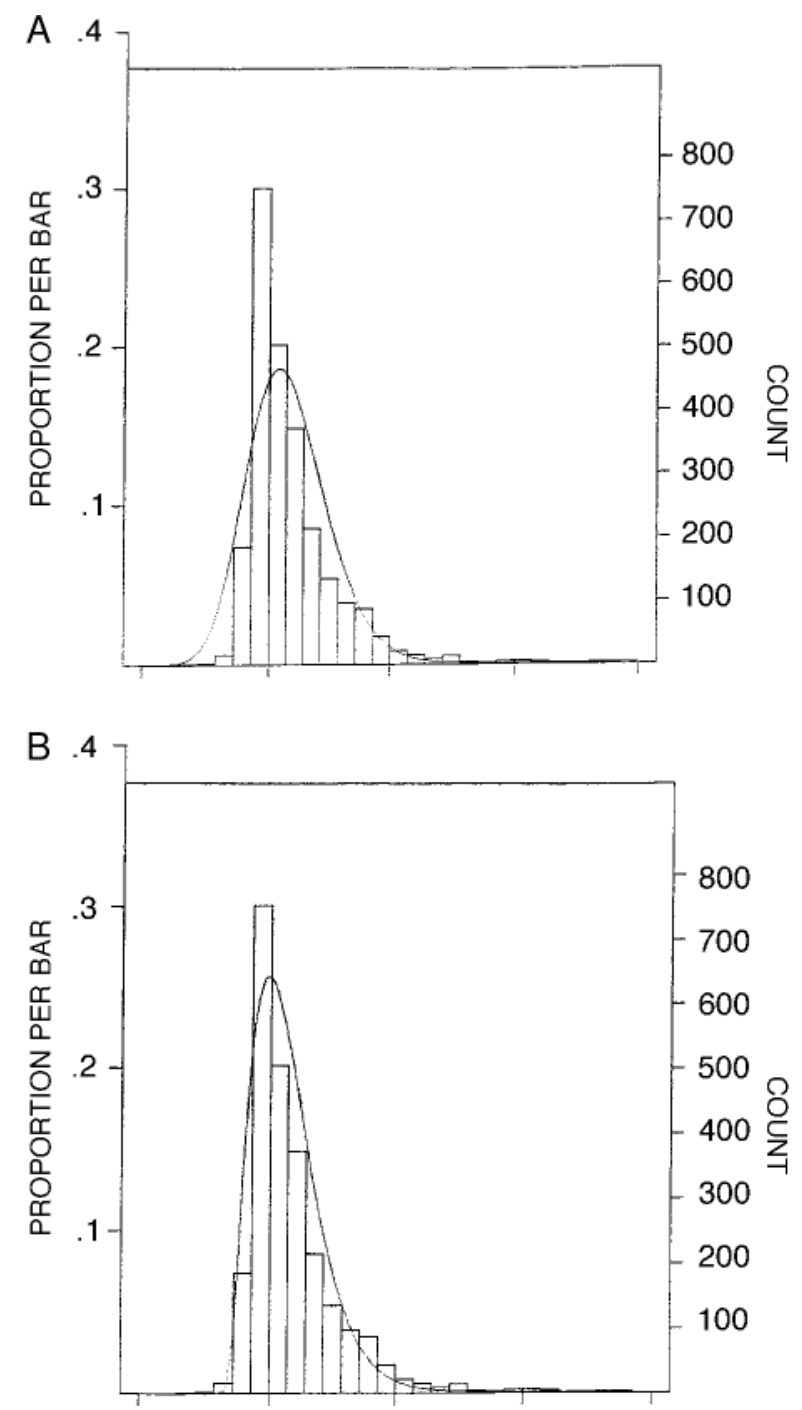

Figure 2. Fit to log-transformed total sample target-present search distribution of (A) 1-distribution and (B) 2-distribution models. 
operate by degrees might always operate together but tend to do so in two preferred combinations (e.g., preponderantly one or preponderantly the other). Fourth, two distinct search processes might exist, both operating on continua of some sort, but one is always in operation and the other is employed only under certain circumstances, yielding a categorical distinction on the basis of the latter's presence or absence. These four alternatives are all abstract, but may suggest ways in which search theorists could construct models that reconcile evidence for the categorical distribution of search slopes with the postulation of single mechanism or continuum models.

A third possible response to the present study's findings might be to question them on statistical grounds. It might be contended that the Gaussian and Gaussian with power transform distributions that commingling analysis uses to model data sets are inappropriate to the analysis of visual search slopes. Although this critique would be somewhat ad hoc, it is certainly possible that the slope distributions that we examined might be very well fitted by a single distribution of a different form. By this account, the superior fit of our 2-distribution models would reflect not the superiority of a distinct process model of visual search but the misspecification of the 1-distribution or continuum model alternative. To our knowledge, no simulation studies have been conducted to assess the vulnerability of commingling analysis to Type I errors of this sort, but, because the analysis does make distributional assumptions, the possibility of error remains open.

Nevertheless, this critique can itself be challenged. First, our commingling analysis findings were consistent across three differently scaled or transformed versions of the data sets, implying some measure of independence from particular distributional assumptions. Second, there is good reason to assume Gaussian or power-transformed Gaussian distributions when modeling in this context, because as MacLean et al. (1976) noted, such distributions are based on many small sources of variation that are more or less independent and combine either additively or multiplicatively. This rather inclusive description would seem to apply to search data, with slopes' being influenced by many factors such as individual differences in processing speed, response style, task type, display characteristics, and many distinct forms of error. Third, there is no obvious a priori reason for proposing any specific non-Gaussian distribution as a basis for modeling visual search, and to choose one of the many more or less obscure alternatives post hoc would be extremely unconstrained. Even so, curve fitting might represent a way forward for developing models of visual search, to the extent that the formal properties of a particularly well-fitting non-Gaussian distribution might offer clues to the nature of the system that could have generated it.

Whatever the implications of the findings for the $a b-$ stract understanding of visual search, they have several more practical implications. First, they suggest that it may not be inappropriate in principle for researchers to divide searches according to the presumed processes involved. The divide may well not be "mythical" after all, and the informal $10 \mathrm{msec} /$ item threshold may in fact be accurately positioned.

Second, the findings suggest that anyone who makes this division should also recognize the serious limitations of doing so. If two distinct search processes exist, and do indeed give rise to the two slope distributions for which our analysis found evidence, these distributions certainly overlap enough for any threshold, however optimal, to yield many incorrect identifications of specific searches. Slopes immediately adjacent to the threshold-say, $9 \mathrm{msec} /$ item and $11 \mathrm{msec} /$ item-do not differ categorically or point with high confidence to distinct types of search, even if such distinct types exist. Although we contend that Wolfe (1998) was probably mistaken in questioning the existence of distinct search processes, and certainly so in taking unimodality to imply a search continuum, he is surely correct in arguing that a binary division of searches cannot be done in a simple, data-driven way. (Overlap rather than unimodality is the issue here, however, since even bimodal distributions generate identification errors unless the component distributions are entirely isolated.) In addition, the threshold certainly cannot be used to sort search types or tasks - as distinct from individual search slopes-into parallel or serial forms, as the findings of this reanalysis indicate that, within each search type, with the possible exception of spatial configuration searches, substantial proportions of slopes fall into both underlying distributions. In short, the reality of the division between distinct search processes can be asserted without at the same time asserting its uncomplicated usefulness for classifying searches, search tasks, or search types.

The findings of the present study are open to several interpretations. However, they suggest that the existence of distinct types of visual search process might have been prematurely ruled out by some theorists. How the evidence for categorically distinct search processes should be integrated with evidence favoring continuum models is a question that awaits further study.

\section{REFERENCES}

Duncan, J., \& Humphries, G. W. (1989). Visual search and stimulus similarity. Psychological Review, 96, 433-458.

Everitt, B. S., \& HAND, D. J. (1981). Finite mixture distributions. New York: Chapman \& Hall.

Maclean, C. J., Morton, N. E., Elston, R. C., \& Yee, S. (1976). Skewness in commingled distributions. Biometrics, 32, 695-699.

MeEHL, P. E. (1995). Bootstraps taxometrics: Solving the classification problem in psychopathology. American Psychologist, 50, 266-275.

Murphy, E. A. (1964). One cause? Many causes? The argument from the bimodal distribution. Journal of Chronic Diseases, 17, 301-324.

Nakayama, K. (1990). The iconic bottleneck and the tenuous link between early visual processing and perception. In C. Blakemore (Ed.), Vision: Coding and efficiency (pp.411-422). Cambridge: Cambridge University Press.

Treisman, A. (1993). The perception of features and objects. In A. Baddeley \& L. Weiskrantz (Eds.), Attention: Selection, awareness, and control (pp. 5-35). Oxford: Oxford University Press, Clarendon Press.

WoLfE, J. M. (1998). What can 1 million trials tell us about visual search? Psychological Science, 9, 33-39.

(Manuscript received May 3, 2000; revision accepted for publication December 15, 2000.) 